\title{
Discovery of technetium- and niobium-rich S stars: The case for bitrinsic stars ${ }^{\star}$
}

\author{
S. Shetye ${ }^{1,2}$, S. Van Eck ${ }^{1}$, S. Goriely ${ }^{1}$, L. Siess ${ }^{1}$, A. Jorissen ${ }^{1}$, A. Escorza ${ }^{2,1}$, and H. Van Winckel ${ }^{2}$ \\ ${ }^{1}$ Institute of Astronomy and Astrophysics (IAA), Université Libre de Bruxelles (ULB), CP 226, Boulevard du Triomphe, \\ 1050 Bruxelles, Belgium \\ e-mail: Shreeya. Shetye@ulb.ac.be \\ 2 Institute of Astronomy, KU Leuven, Celestijnenlaan 200D, 3001 Leuven, Belgium
}

Received 10 January 2020 / Accepted 18 February 2020

\begin{abstract}
Context. S stars are late-type giants with overabundances of $s$-process elements. They come in two flavors depending on the presence or lack of presence of technetium (Tc), an element without stable isotopes. Intrinsic S stars are Tc-rich and genuine asymptotic giant branch (AGB) stars, while extrinsic S stars owe their s-process over abundances to the pollution from a former AGB companion, which is now a white dwarf (WD). In addition to Tc, another distinctive feature between intrinsic and extrinsic S stars is the overabundance of niobium $(\mathrm{Nb})$ in the latter class. Indeed, since the mass transfer occurred long ago, ${ }^{93} \mathrm{Zr}$ had time to decay into the only stable isotope of $\mathrm{Nb},{ }^{93} \mathrm{Nb}$, causing its overabundance.

Aims. We discuss the case of the $\mathrm{S}$ stars $\mathrm{BD}+79^{\circ} 156$ and $o^{1}$ Ori, whose specificity lies in sharing the distinctive features of both intrinsic and extrinsic $\mathrm{S}$ stars, namely the presence of Tc along with a $\mathrm{Nb}$ overabundance.

Methods. We used high-resolution HERMES optical spectra, MARCS model atmospheres of S stars, Gaia DR2 parallaxes, and STAREVOL evolutionary tracks to determine the stellar parameters and chemical abundances of the two $\mathrm{S}$ stars, and to locate them in the Hertzsprung-Russell (HR) diagram.

Results. $\mathrm{BD}+79^{\circ} 156$ is the first clear case of a bitrinsic star, that is, a doubly $s$-process-enriched object, first through mass transfer in a binary system and then through internal nucleosynthesis that is responsible for the Tc-enrichment in BD $+79^{\circ} 156$, which must, therefore, have reached the AGB phase of its evolution. This hybrid nature of the s-process pattern in $\mathrm{BD}+79^{\circ} 156$ is supported by its binary nature and its location in the HR diagram that is just beyond the onset of the third dredge-up on the AGB. The Tc-rich, binary S-star $o^{1}$ Ori with a WD companion was another long-standing candidate for a similar hybrid $s$-process enrichment. However, the marginal overabundance of $\mathrm{Nb}$ derived in $o^{1}$ Ori does not allow one to trace evidence of large amounts of pollution coming from the AGB progenitor of its current WD companion unambiguously. As a side product, the current study offers a new way of detecting binary AGB stars with WD companions by identifying their Tc-rich nature along with a $\mathrm{Nb}$ overabundance.
\end{abstract}

Key words. stars: AGB and post-AGB - stars: abundances - nuclear reactions, nucleosynthesis, abundances - stars: interiors Hertzsprung-Russell and C-M diagrams

\section{Introduction}

$\mathrm{S}$ stars are late-type giants with molecular bands of $\mathrm{ZrO}$ and $\mathrm{TiO}$, which are the most characteristic spectroscopic feature (Merrill 1922). They are transition objects between M-type stars and carbon stars on the asymptotic giant branch (AGB) as their carbon/oxygen $(\mathrm{C} / \mathrm{O})$ ratio is in the range $0.5 \leq \mathrm{C} / \mathrm{O}<1$ (Iben \& Renzini 1983; Scalo \& Ross 1976; Van Eck et al. 2017). The strong $\mathrm{ZrO}$ bands actually reflect overabundances of slow neutron-capture ( $s$-process) elements in their spectra (Keenan 1954; Smith \& Lambert 1990).

$\mathrm{S}$ stars come in two flavors. Intrinsic $\mathrm{S}$ stars are defined as Tcenriched $\mathrm{S}$ stars. Since $\mathrm{Tc}$ is an element with no stable isotopes and its ${ }^{99} \mathrm{Tc}$ isotope produced by the $s$-process has a half-life $T_{1 / 2}$ of $2.1 \times 10^{5} \mathrm{yr}$, its detection indicates that this element is currently synthesized by the star located on the AGB, which is undergoing

\footnotetext{
* Based on observations made with the Mercator Telescope, operated on the Island of La Palma by the Flemish Community, at the Spanish Observatorio del Roque de los Muchachos of the Instituto de Astrofísica de Canarias.
}

third-dredge-up (TDU) episodes. On the other hand, in extrinsic $\mathrm{S}$ stars, Tc was brought long ago by a former AGB companion through mass transfer. Because the time that elapsed since this mass-transfer episode is most likely much longer than the ${ }^{99} \mathrm{Tc}$ half-life, Tc had time to decay in ${ }^{99} \mathrm{Ru}$ so that Tc lines are thus absent from the spectrum. Extrinsic S stars that are not yet on the thermally-pulsing-(TP-) AGB can thus not produce $s$-process elements themselves (Van Eck et al. 1998; Shetye et al. 2018). They have all been shown to be binaries (Jorissen et al. 1998, 2019).

The isotope ${ }^{93} \mathrm{Zr}$ has a half-life $T_{1 / 2}=1.53 \times 10^{6} \mathrm{yr}$, and it is another radio-isotope for which the abundance corroborates the above scenario. In extrinsic $\mathrm{S}$ stars, this isotope had time to decay into ${ }^{93} \mathrm{Nb}$, which is the only stable $\mathrm{Nb}$ isotope. On the contrary, in intrinsic $\mathrm{S}$ stars, ${ }^{93} \mathrm{Zr}$ is still alive, meaning that the $\mathrm{Nb}$ abundance is still low (i.e., with a pre-s-process, solar-scaled abundance; Neyskens et al. 2015).

In the current work, through abundance analyses of BD + $79^{\circ} 156$ and $o^{1}$ Ori, we present a new type of S star, showing signatures of both intrinsic and extrinsic S stars. We label them "bitrinsic" S stars. A bitrinsic star can be defined as a star having 
passed through a double pollution in $s$-process elements, occurring first when its companion is on the TP-AGB, which pollutes it through mass transfer, and second when its evolves on the TPAGB and enriches its envelope by dredge-ups. These two events can only be traced by measuring $\mathrm{Nb}$ (enhanced in mass-transfer systems) and Tc (enhanced in TP-AGB objects) abundances. This new class of $\mathrm{S}$ stars has been unraveled thanks to the availability of high-resolution spectra and of Gaia data-release 2 (GDR2) parallaxes (Gaia Collaboration 2018). In Sect. 2, we describe the observations, the detection of Tc, and discuss the binary nature of the studied systems. In Sects. 3-5, we present the atmospheric parameters, surface abundances, and location in the HertzsprungRussell (HR) diagram, which confirms the genuine AGB evolutionary status of these bitrinsic candidates. Abundances are then compared to STAREVOL nucleosynthesis predictions in Sect. 6. Section 7 summarizes our analysis.

\section{Searching for bitrinsic S stars}

Among the S stars from the General Catalogue of Galactic S Stars (Stephenson 1984, CGSS), we selected a sample of S stars that fulfill the following conditions due to quality concerns: First, stars with $\sigma_{\varpi} / \varpi \leq 0.3$, where $\varpi$ is the Gaia parallax and $\sigma_{\varpi}$ is its error. Second, stars with declinations north of $-30^{\circ}$ and $V$ magnitudes brighter than $11 \mathrm{mag}$ are to be observed with the HERMES high-resolution spectrograph ${ }^{1}$ (Raskin et al. 2011). Third, stars with HERMES spectra having a signal-to-noise ratio $(\mathrm{S} / \mathrm{N})$ of 50 in the $V$ band since a high $\mathrm{S} / \mathrm{N}$ is required to ensure the Tc-line detectability (Van Eck et al. 2017; Shetye et al. 2018).

Out of this sample, we found two candidates, namely $\mathrm{BD}+79^{\circ} 156$ and $o^{1}$ Ori, that show the presence of Tc lines and $\mathrm{Nb}$ overabundances. The following sections present the detailed analysis of these stars. The analysis of the rest of this sample is presented elsewhere (Shetye et al. 2018, 2019) and in a forthcoming paper (Shetye et al., in prep.).

\subsection{Technetium detection}

The detection of $\mathrm{Tc}$ is made by using the three TcI resonance lines located at $4238.19 \AA, 4262.27 \AA$, and $4297.06 \AA$. Based on medium-resolution spectra, Jorissen et al. (1993) classified $\mathrm{BD}+79^{\circ} 156$ as a Tc-deficient object. A subsequent study of infrared colors (which constitute an indirect classification criterion since intrinsic stars generally exhibit infrared excesses indicative of mass loss; Jorissen et al. 1993; Van Eck \& Jorissen 2000) by Yang et al. (2006) and Otto et al. (2011) classified BD $+79^{\circ} 156$ as well as an extrinsic S star. Regarding $o^{1}$ Ori, Wallerstein \& Dominy (1988) and Smith \& Lambert (1988) derived its Tc abundance from medium-resolution spectra and thus classified it as intrinsic (Tc-rich). This claim was confirmed by Wang \& Chen (2002) from near-infrared observations.

However, as shown in Fig. 1 based on high-resolution HERMES spectra, absorption features are clearly present at the wavelengths of the three ultraviolet (UV) Tc lines for the two stars $o^{1}$ Ori and $\mathrm{BD}+79^{\circ} 156$. The spectra of $\mathrm{BD}+79^{\circ} 156, o^{1}$ Ori, and a reference Tc-rich S star (V915 Aql) are in sharp contrast with those of the reference Tc-poor S star HD 233158, which are shown for comparison purposes. From our analysis of highresolution spectra, we thus reclassify $\mathrm{BD}+79^{\circ} 156$ as a Tc-rich $\mathrm{S}$ star (Fig. 1).

\footnotetext{
1 HERMES is a high-resolution $(R=86000)$ spectrograph mounted on the Mercator Telescope at the Roque de los Muchachos Observatory, La Palma (Canary Islands).
}
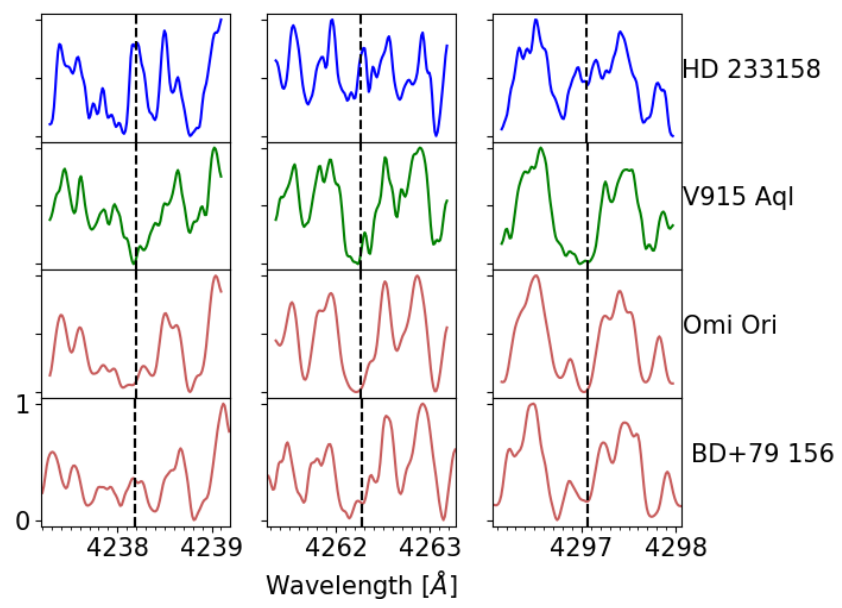

Fig. 1. Spectral region around the three $(4238.19,4262.27$, and 4297.06 Å) ultraviolet Tc I lines for Tc-poor (HD 233158, blue spectrum in the top panels) and Tc-rich (V915 Aql, green spectrum in the second panels from the top) S stars from Shetye et al. (2018) and for the bitrinsic candidates $o^{1}$ Ori and $\mathrm{BD}+79^{\circ} 156$ (bottom panels). The spectra have been arbitrarily normalized and binned by a factor of 1.5 to increase the $\mathrm{S} / \mathrm{N}$.

\subsection{Binarity}

The binary nature of $o^{1}$ Ori was first assessed by the discovery of a white dwarf (WD) companion in International Ultraviolet Explorer (IUE) data, with spectral type DA3 and $T_{\text {eff }}=22000 \mathrm{~K}$ (Ake \& Johnson 1988). Prior to that discovery, Peery (1986) had reported the detection of emission lines from ionized elements in IUE spectra of $o^{1}$ Ori, but he could not decide whether these lines originate from the giant star due to flares, for instance, or if they were attributable to the presence of a hot subluminous companion. $o^{1}$ Ori has since been an enigma because Tc-rich $\mathrm{S}$ stars ought not to have a WD companion, in contrast to Tcpoor stars. But it has remained unclear until now whether this WD had polluted its companion with heavy elements while on the TP-AGB, or in other words, whether this star was extrinsic before turning intrinsic. This question is addressed in Sect. 6 .

The derivation of the orbital elements of the $o^{1}$ Ori system has turned out to be very difficult (Jorissen et al. 2019) because the radial-velocity data show a very limited excursion of 2 to $3 \mathrm{~km} \mathrm{~s}^{-1}$ with some superimposed radial-velocity jitter. A tentative period of $575 \mathrm{~d}$ and a mass function of $(2.7 \pm 0.2) \times$ $10^{-5} M_{\odot}$ was obtained by Jorissen et al. (2019), which is suggestive of an orbit seen almost pole-on $\left(i=4.7^{\circ} \pm 1^{\circ}\right)$.

$\mathrm{BD}+79^{\circ} 156$ is a confirmed binary with an orbital period of 10931 days, an eccentricity of 0.461 , and a mass function of $0.025 M_{\odot}$, which is compatible with a WD companion (Van der Swaelmen et al. 2017; Jorissen et al. 2019). An additional confirmation of the presence of a WD companion around $\mathrm{BD}+79^{\circ} 156$ (and $o^{1}$ Ori) comes from the UV excess seen in their spectral energy distribution (SED), which is well visible when comparing photometric observations with model SED (Fig. A.1). The binarity criteria proposed by Ortiz \& Guerrero (2016) based on the GALEX near-UV observed to predicted flux ratios confirm that both $o^{1}$ Ori and BD $+79^{\circ} 156$ are in a binary system.

\section{Derivation of the atmospheric parameters}

The stellar parameters impacting the atmospheric thermal structure of $\mathrm{S}$ stars are $T_{\text {eff }}, \log g,[\mathrm{Fe} / \mathrm{H}], \mathrm{C} / \mathrm{O}$, and [s/Fe]. To disentangle this multidimensional parameter space, we followed the 
same methodology as described in Shetye et al. (2018). Briefly, this method performs a $\chi^{2}$ minimization search over several spectral windows, comparing the observed spectrum with synthetic MARCS model atmospheres of S stars (Van Eck et al. 2017). The best-fitting model is the one with the lowest $\chi^{2}$ value and the corresponding parameters constitute our first estimates.

The luminosity derived from the Gaia parallax and the first estimate of $T_{\text {eff }}$ from the $\chi^{2}$ fitting tool allowed us to locate the star in the HR diagram. From this, we derived the stellar mass and hence the surface gravity by comparing this position in the HR diagram with STAREVOL evolutionary tracks. We then performed an iteration on $\log g$ and on the stellar parameters, as described in Shetye et al. (2018), in order to obtain a $\log g$ value that is consistent with the mass inferred from the location in the $\mathrm{HR}$ diagram. The mass that we derived is $\approx 2 M_{\odot}$ for $\mathrm{BD}+79^{\circ} 156$ and $1.5 M_{\odot}$ for $o^{1}$ Ori. The final list of stellar parameters is presented in Table $1^{2}$.

\section{Abundance determination}

We used the radiative transfer code TURBOSPECTRUM (Alvarez \& Plez 1998; Plez 2012) to generate synthetic spectra from the MARCS model atmospheres of the S stars with the atmospheric parameters, which were determined as described in Sect. 3. We used the $\mathrm{C} / \mathrm{O}$ estimates from the $\chi^{2}$ spectral fitting tool and checked whether these values reproduce the observed spectra satisfactorily in the $\mathrm{CH}$ bands around $4200 \AA-4300 \AA$. Because atomic oxygen lines are poorly reproduced in S-type stars, we adopted the solar oxygen abundance from Asplund et al. (2009), which is scaled to the star metallicity. The nitrogen abundance was derived using the CN bands at $7900 \AA-8100 \AA$.

The metallicity was determined using at least ten isolated $\mathrm{Fe}$ lines in the region from $7350 \AA$ to $8730 \AA$. The derived $[\mathrm{Fe} / \mathrm{H}]$ and its standard deviation are provided in Table 1 . The $s$-process abundances are listed in Table A.1. The $\mathrm{Zr}$ abundance was derived using two Zr I lines at $7819.37 \AA$ and $7849.37 \AA$ with transition probabilities from laboratory measurements (Biémont et al. 1981). We used three $\mathrm{Nb}$ I lines located at $5189.186 \AA$, $5271.524 \AA$, and $5350.722 \AA$ to derive the $\mathrm{Nb}$ abundances in the sample stars. Details about the $\mathrm{Fe}, \mathrm{Zr}$, and $\mathrm{Nb}$ lines can be found in Table A.2. The Tc abundance was derived by only using the Tc line at $4262.27 \AA$. The other $s$-process abundances were also determined with the goal of obtaining a full $s$-process abundance profile (presented in Table A.1). A systematic analysis of these abundances along with those of the other Tc-rich S stars is deferred to a forthcoming paper.

For abundance uncertainties that are induced by the uncertainties on the atmospheric parameters, we used those of V915 Aql from Shetye et al. (2018) since the atmospheric parameters of V915 Aql $\left(T_{\text {eff }}=3400 \mathrm{~K}, \log g=0.0,[\mathrm{Fe} / \mathrm{H}]=-0.5, \mathrm{C} / \mathrm{O}=0.75\right.$, $[\mathrm{s} / \mathrm{Fe}]=0.0)$ are appropriate matches for those of our sample stars. We computed the total abundance uncertainty $\left(\sigma_{[\mathrm{X} / \mathrm{Fe}]}\right.$ in Table A.1) by quadratically adding the elemental standard deviation due to line-to-line scatter, the atmospheric parameterinduced uncertainties as estimated for V915 Aql, and a 0.1 dex uncertainty related to the continuum placement. When only one spectral line was used to derive the abundance of a given element, a standard line-to-line scatter of 0.1 dex was adopted. The abundances and their uncertainties are listed in Table A.1.

\footnotetext{
2 We note that the atmospheric parameters of $o^{1}$ Ori quoted in Jorissen et al. (2019) are different than the ones used in this study. This is because $\log g$ iterations were not performed in Jorissen et al. (2019)
}

Table 1. Main properties of the bitrinsic S stars.

\begin{tabular}{lcc}
\hline \hline & $o^{1}$ Ori & BD $+79^{\circ} 156$ \\
\hline \multicolumn{2}{c}{ Basic data } \\
\hline Sp. type & M3SIII & $\mathrm{S} 4 / 2$ \\
$V(\mathrm{mag})$ & 4.72 & 9.55 \\
$K(\mathrm{mag})$ & 0.48 & 5.67 \\
$\varpi \pm \sigma_{\varpi}(\mathrm{mas})$ & $6.16 \pm 0.41$ & $0.57 \pm 0.03$ \\
$E_{B-V}$ & 0.07 & 0.26 \\
$\mathrm{BC} \mathrm{C}_{K}(\mathrm{mag})$ & 2.91 & 2.85 \\
\hline \multicolumn{3}{c}{ Atmospheric parameters } \\
\hline$T_{\text {eff }}(\mathrm{K})$ & 3500 & 3600 \\
& $(3500-3600)$ & $(3600-3700)$ \\
$L\left(L_{\odot}\right)$ & 2700 & 2800 \\
& $(2300-3100)$ & $(2700-2900)$ \\
$\log g$ & 1 & 1 \\
& $(0-2)$ & $(1-3)$ \\
$M\left(M_{\odot}\right)$ & 1.5 & 2.0 \\
$\mathrm{C} / \mathrm{O}$ & $(1.3-2)$ & $(1.5-2.5)$ \\
& 0.75 & 0.50 \\
{$[\mathrm{~s} / \mathrm{Fe}]$} & $(0.50-0.90)$ & $(0.50-0.75)$ \\
& 0 & 1 \\
{$[\mathrm{Fe} / \mathrm{H}]$} & $(0-1)$ & $(1-1)$ \\
$\sigma_{[\mathrm{Fe} / \mathrm{H}]}$ & -0.28 & -0.16 \\
\hline
\end{tabular}

Notes. $V$ and $K$ magnitudes have been extracted from the SIMBAD Astronomical Database (Wenger et al. 2000). The parallax $\varpi$ and its uncertainty $\sigma_{\varpi}$ are from GDR2 (Gaia Collaboration 2018). The reddenning $E_{B-V}$ has been obtained from the Gontcharov (2012) extinction maps. $\mathrm{BC}_{K}$ is the bolometric correction in the $K$ band as computed from the MARCS model atmospheres. Atmospheric parameters for bitrinsic stars are also given (see Sect. 3). Values between parentheses correspond to the parameter range provided by the $\chi^{2}$ fitting. The metallicities $[\mathrm{Fe} / \mathrm{H}]$ have been obtained from the $\mathrm{Fe}$ abundance analyses. The standard deviation $\sigma_{[\mathrm{Fe} / \mathrm{H}]}$ represents the line-to-line scatter on $[\mathrm{Fe} / \mathrm{H}]$. The masses have been derived from the locations of the stars in the HR diagram compared to the STAREVOL tracks.

\section{Location of the bitrinsic S stars in the HR diagram}

In order to infer the evolutionary status of S stars, we located them in the HR diagram by using both the effective temperature derived as described in Sect. 3 and luminosities derived using the Gaia DR2 parallaxes. We compare these positions in the HR diagram with the STAREVOL evolutionary tracks of the corresponding metallicities (Fig. 2). $o^{1}$ Ori and $\mathrm{BD}+79^{\circ} 156$ are both located above the black dashed line that marks the predicted onset of TDU in the HR diagram. This confirms that they are TP-AGB stars. The fact that they are not very evolved on the TP-AGB is consistent with their moderate $s$-process abundances, relatively low $\mathrm{C} / \mathrm{O}$ ratios, and absence of infrared excesses.

\section{Comparison with STAREVOL nucleosynthesis predictions}

The $\mathrm{Tc}$ and $\mathrm{Nb}$ abundances are inversely correlated and a clear segregation between intrinsic and extrinsic $\mathrm{S}$ stars is expected in the $\mathrm{Zr}-\mathrm{Nb}$ plane, as seen in Fig. 3. We now compare the measured abundances with the nucleosynthesis predictions from the STAREVOL code (Siess \& Arnould 2008; Goriely \& Siess 2018). The partial mixing of protons in the C-rich layers, which is responsible for the $s$-process nucleosynthesis, is described by 


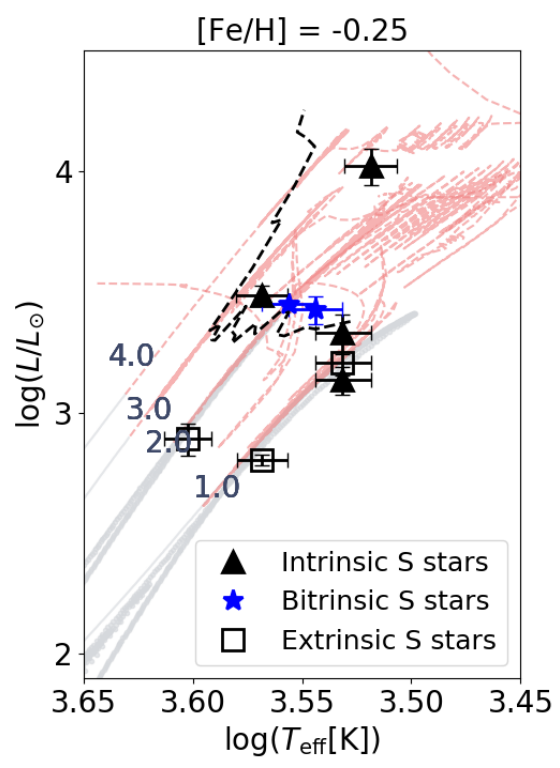

Fig. 2. HR diagram of intrinsic (filled triangles) $S$ stars from Shetye et al. (2018, 2019), bitrinsic S stars (blue stars), and extrinsic (open squares) S stars from Shetye et al. (2018) along with the STAREVOL evolutionary tracks corresponding to the closest grid metallicity. The red giant branch is represented by the gray dots, whereas the red dashed line corresponds to the AGB tracks. The black dashed line marks the onset of TDU.

a diffusive equation with the mixing parameters $f_{\text {over }}=0.1$, $D_{\text {min }}=10^{9} \mathrm{~cm}^{2} \mathrm{~s}^{-1}$, and $p=5$, where $f_{\text {over }}$ controls the extent of the mixing, $D_{\min }$ is the value of the diffusion coefficient at the innermost boundary of the diffusive region, and $p$ is an additional free parameter describing the shape of the diffusion profile. Other sets of mixing parameters could be considered (Shetye et al. 2019). All details can be found in Goriely \& Siess (2018) and references therein.

In Fig. 3, we present the predictions for intrinsic and extrinsic stars with masses 2 and $3 M_{\odot}$, and $[\mathrm{Fe} / \mathrm{H}]=0.0$ and -0.2 . Our sample stars $\mathrm{BD}+79^{\circ} 156$ and $o^{1}$ Ori are both clearly Tc-rich. According to the $\mathrm{Nb} / \mathrm{Tc}$ anticorrelation, they should thus not be enriched in $\mathrm{Nb}$, contrary to what is observed in the $([\mathrm{Zr} / \mathrm{Fe}]$, $[\mathrm{Nb} / \mathrm{Fe}]$ ) plane (Fig. 3). BD $+79^{\circ} 156$ is found within the extrinsic stars $([\mathrm{Zr} / \mathrm{Fe}]=0.84,[\mathrm{Nb} / \mathrm{Fe}]=0.81)$ along the predicted theoretical trend for extrinsic $\mathrm{S}$ stars. Its $[\mathrm{Zr} / \mathrm{Nb}]$ ratio is close to unity, which indicates that ${ }^{93} \mathrm{Zr}$ has decayed and that a masstransfer episode from a former AGB star has taken place. However, because $\mathrm{BD}+79^{\circ} 156$ is enriched in Tc as well, it must currently also be an AGB star. The bitrinsic star BD $+79^{\circ} 156$ thus constitutes an example of dual-enrichment events (one extrinsic, one intrinsic).

In $o^{1}$ Ori, $\mathrm{Nb}$ is overabundant but to a much lesser extent $([\mathrm{Nb} / \mathrm{Fe}]=0.12,[\mathrm{Zr} / \mathrm{Fe}]=0.15)$. Its position in Fig. 3 is less conclusive. However, its $[\mathrm{Zr} / \mathrm{Nb}]$ ratio is close to unity as in the case of $\mathrm{BD}+79^{\circ} 156$. Besides, a WD companion has been detected for $o^{1}$ Ori (Sect. 2.2 and Ake \& Johnson 1988), strengthening the case for it being a bitrinsic object.

To account for the surface composition of $\mathrm{BD}+79^{\circ} 156$, we computed the evolution of a $2 M_{\odot}$ star with metallicity $[\mathrm{Fe} / \mathrm{H}]=-0.3$, which is representative of the observed star. This model accounts for the production of Tc. To account for the $\mathrm{Nb}$ overabundance, we then assume it to be due to the ${ }^{93} \mathrm{Zr}$ pollution from a former $3 M_{\odot}$ AGB companion, which is now a dim WD. The composition of the current star is then the result of the enrichment coming from its companion through mass trans-

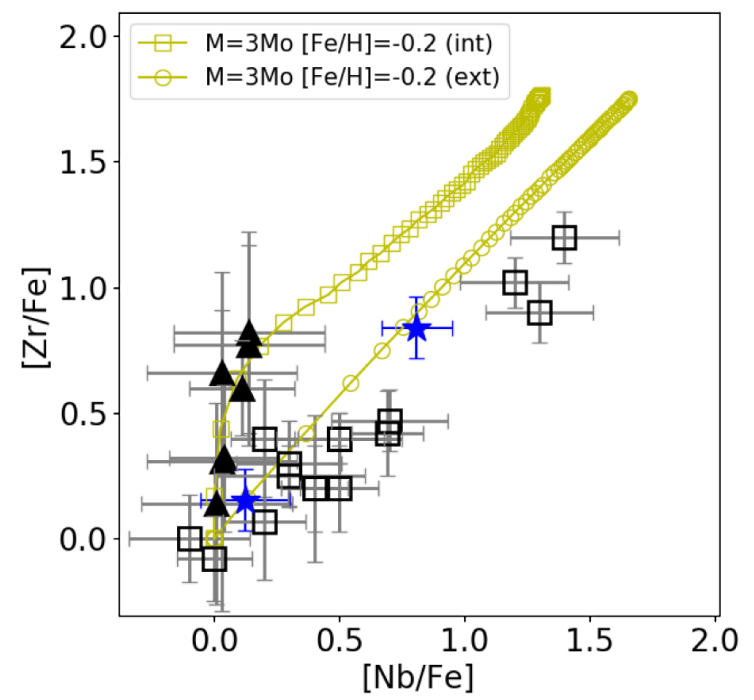

Fig. 3. $[\mathrm{Zr} / \mathrm{Fe}]$ vs $[\mathrm{Nb} / \mathrm{Fe}]$ ratios for the sample stars (blue star symbols), as compared with intrinsic (triangles) and extrinsic (open squares) $\mathrm{S}$ stars from Shetye et al. $(2018,2019)$. The yellow-green curves are the nucleosynthesis predictions from STAREVOL for $3 M_{\odot},[\mathrm{Fe} / \mathrm{H}]=-0.2$ models. Each symbol along these curves represents a thermal pulse. The $2 M_{\odot}$ models completely overlap the $3 M_{\odot}$ ones, but they only reach up to $[\mathrm{Zr} / \mathrm{Fe}] \sim 1.3$.

fer as well as from its own TDU episodes. By adjusting the amount of material diluted in the envelope of the $2 M_{\odot}$ star from its companion and the intrinsic surface enrichment through TDU episodes (i.e., the number of thermal pulses encountered so far), we can reproduce the observed abundances fairly well, as shown in Fig. 4. Within this nonunique parameter set (describing mixing and stellar masses), a non-negligible $10 \%$ dilution factor from $\mathrm{BD}+79^{\circ} 156^{\prime}$ 's companion wind is needed to explain the high $\mathrm{Nb}$ abundance found at its surface. We conclude that for $\mathrm{BD}+79^{\circ} 156$, a double pollution event is necessary to explain the abundance pattern. It is found not only to reproduce the measured Tc and $\mathrm{Nb}$ abundances but also those of light and heavy $s$-process elements. This conclusion holds independent of the uncertainties affecting the mixing parameters in the description of the diffusion processes. No other elements besides $\mathrm{Nb}$ and Tc can unambiguously point out toward such a double pollution mechanism.

Concerning $o^{1}$ Ori, this system has been considered to be a prototype bitrinsic star for a very long time (Ake \& Johnson 1988; Jorissen et al. 1993, 2019). As shown in the bottom panel of Fig. 4 where we compare the abundance profile of $o^{1}$ Ori with the predictions of intrinsic and extrinsic stars, a pollution of $s$-process elements by a binary companion may not be needed because of the slight overabundance in $\mathrm{Nb}$. Hence $o^{1}$ Ori is actually a star with only a marginal bitrinsic character. We support the claims of Ake \& Johnson (1988) that, though the existence of a WD companion is very clear for $o^{1}$ Ori, there are no signatures in the chemical abundances that would need an explanation other than the occurrence of intrinsic $s$-process nucleosynthesis followed by TDUs on the AGB. We thus regard $o^{1}$ Ori as a Tc-rich $\mathrm{S}$ star with a WD companion, but there is no clear evidence of a large pollution coming from the AGB progenitor of this companion.

\section{Conclusions}

$\mathrm{S}$ stars of a new type have been uncovered, bearing characteristics of both intrinsic and extrinsic S stars, and they are referred to as bitrinsic stars. The intriguing hybrid classification as both 


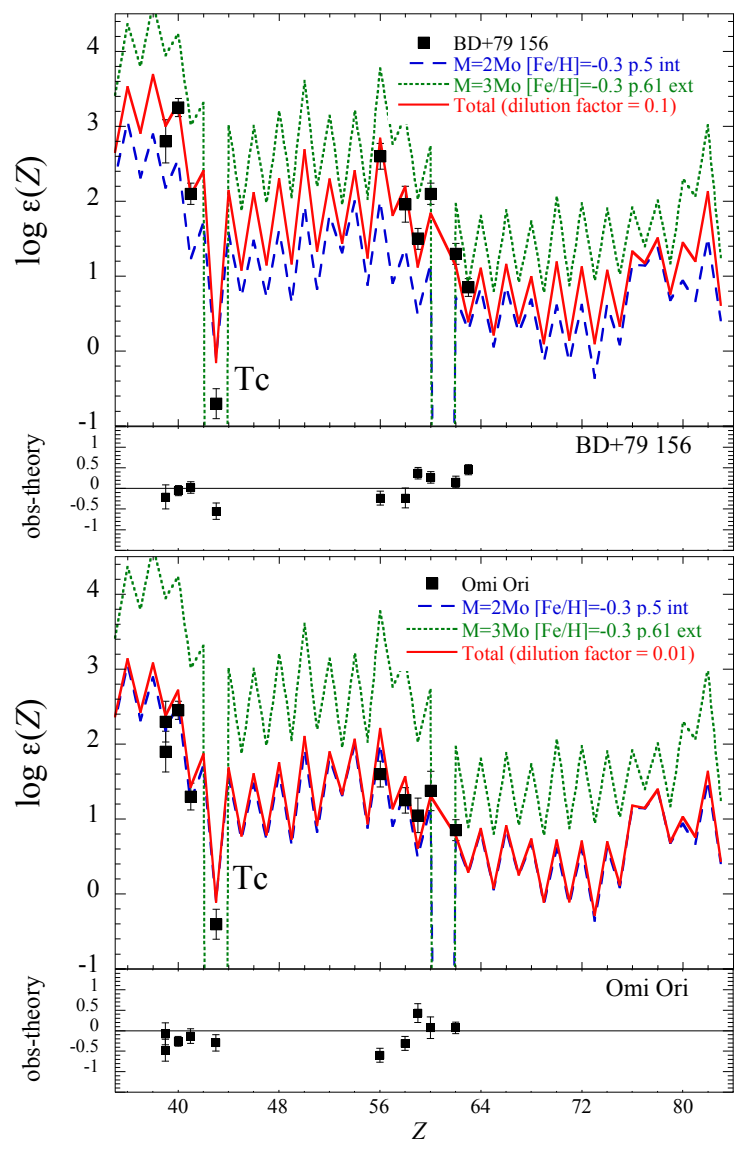

Fig. 4. Comparison of the observed and predicted surface abundances for $\mathrm{BD}+79^{\circ} 156$ (top panel) and $o^{1}$ Ori (bottom panel) in unit of $\log \epsilon$. The best fit (solid red curves) results from the dilution of the yields from a $3 M_{\odot},[\mathrm{Fe} / \mathrm{H}]=-0.3$ model within the envelope of a $2 M_{\odot}$ $[\mathrm{Fe} / \mathrm{H}]=-0.3$ intrinsic AGB star after it has experienced five thermal pulses. In both panels, "p.5 int" means that five pulses were involved in the intrinsic contribution whereas "p61 ext" means that 61 pulses occurred in the companion star (considering mass transfer at the end of the TP-AGB phase of the companion).

intrinsic (because of Tc detection) and extrinsic (because of $\mathrm{Nb}$ overabundance and WD companion detection) can be understood if we identify these stars with objects formerly polluted by an AGB companion, which now evolve on the TP-AGB. Though such objects were expected to exist, none of them had been identified until now because of the lack of appropriate diagnostics. Thanks to a careful analysis of S-type stars involving atmospheric parameters and abundance determination using HERMES highresolution spectra, we could identify one clear case $\left(\mathrm{BD}+79^{\circ} 156\right)$ of such doubly-polluted objects and one tentative case $\left(o^{1}\right.$ Ori).

These objects are interesting in several respects. First, if appropriate statistics of bitrinsic stars were available, this would put interesting constraints on binary-synthesis evolution models because the number of bitrinsic star depends, among other things, on the radius evolution during the RGB and AGB phases, which determines whether and when a Roche-lobe overflow occurs, causing the disappearance of systems that would have become bitrinsic objects (Escorza et al. 2020). A second constraint concerns the component masses: in order to exhibit $s$ process overabundances at their surface, AGB stars must have an initial mass that is larger than $1 M_{\odot}$ (as recently shown by Shetye et al. 2019) and smaller than $4-5 M_{\odot}$, above which the radiative $s$-process production is significantly reduced (Goriely
\& Siess 2004). Therefore, in the case of bitrinsic systems, both the primary and secondary components should satisfy this condition (along with $M_{1}>M_{2}$ ). Third, spectroscopic binaries involving AGB stars are difficult to find because their radial-velocity variations are blurred by strong imprints from envelope pulsations. $\mathrm{Tc}$ and $\mathrm{Nb}$ diagnostics thus offer a direct spectroscopic way to prove the binarity of an AGB star, without resorting to a time-consuming radial-velocity monitoring. Fourth, bitrinsic stars should exist among other stellar classes as well, and in the future they should be sought out for among carbon stars and possibly among lower-metallicity carbon-enriched objects.

Acknowledgements. This research has been funded by the Belgian Science Policy Office under contract BR/143/A2/STARLAB. S.V.E. thanks Fondation ULB for its support. Based on observations obtained with the HERMES spectrograph, which is supported by the Research Foundation - Flanders (FWO), Belgium, the Research Council of KU Leuven, Belgium, the Fonds National de la Recherche Scientifique (F.R.S.-FNRS), Belgium, the Royal Observatory of Belgium, the Observatoire de Genève, Switzerland and the Thüringer Landessternwarte Tautenburg, Germany. LS and SG are senior FNRS research associates. AE acknowledges the support of FWO under contract ZKD1501-00-W01.

\section{References}

Ake, T. B., \& Johnson, H. R. 1988, ApJ, 327, 214

Alvarez, R., \& Plez, B. 1998, A\&A, 330, 1109

Asplund, M., Grevesse, N., Sauval, A. J., \& Scott, P. 2009, ARA\&A, 47, 481

Biémont, E., Grevesse, N., Hannaford, P., \& Lowe, R. M. 1981, ApJ, 248, 867

Duquette, D. W., \& Lawler, J. E. 1982, Phys. Rev. A, 26, 330

Escorza, A., Siess, L., Jorissen, A., \& Van Winckel, H. 2020, A\&A, submitted

Gaia Collaboration (Brown, A. G. A., et al.) 2018, A\&A, 616, A1

Gontcharov, G. A. 2012, Astron. Lett., 38, 87

Goriely, S., \& Siess, L. 2004, A\&A, 421, L25

Goriely, S., \& Siess, L. 2018, A\&A, 609, A29

Iben, Jr., I., \& Renzini, A. 1983, ARA\&A, 21, 271

Jorissen, A., Frayer, D. T., Johnson, H. R., Mayor, M., \& Smith, V. V. 1993, A\&A, 271, 463

Jorissen, A., Van Eck, S., Mayor, M., \& Udry, S. 1998, A\&A, 332, 877

Jorissen, A., Boffin, H. M. J., Karinkuzhi, D., et al. 2019, A\&A, 626, A127

Keenan, P. C. 1954, ApJ, 120, 484

Kurucz, R. L. 2007, Robert L. Kurucz On-line Database of Observed and Predicted Atomic Transitions

Martin, G., Fuhr, J., \& Wiese, W. 1988, J. Phys. Chem. Ref. Data Suppl., 17

Merrill, P. W. 1922, ApJ, 56, 457

Nave, G., Johansson, S., Learner, R. C. M., Thorne, A. P., \& Brault, J. W. 1994, ApJS, 94, 221

Neyskens, P., Van Eck, S., Jorissen, A., et al. 2015, Nature, 517, 174

O'Brian, T. R., Wickliffe, M. E., Lawler, J. E., Whaling, W., \& Brault, J. W. 1991, J. Opt. Soc. Am. B Opt. Phys., 8, 1185

Ortiz, R., \& Guerrero, M. A. 2016, MNRAS, 461, 3036

Otto, E., Green, P. J., \& Gray, R. O. 2011, ApJS, 196, 5

Peery, Jr., B. F. 1986, ESA Spec. Publ., 263, 117

Plez, B. 2012, Astrophysics Source Code Library [record ascl:1205.004]

Raskin, G., van Winckel, H., Hensberge, H., et al. 2011, A\&A, 526, A69

Scalo, J. M., \& Ross, J. E. 1976, A\&A, 48, 219

Shetye, S., Van Eck, S., Jorissen, A., et al. 2018, A\&A, 620, A148

Shetye, S., Goriely, S., Siess, L., et al. 2019, A\&A, 625, L1

Siess, L., \& Arnould, M. 2008, A\&A, 489, 395

Smith, V. V., \& Lambert, D. L. 1988, ApJ, 333, 219

Smith, V. V., \& Lambert, D. L. 1990, ApJS, 72, 387

Stephenson, C. B. 1984, Publications of the Warner and Swasey Observatory, 3, 1

Van der Swaelmen, M., Boffin, H. M. J., Jorissen, A., \& Van Eck, S. 2017, A\&A, 597, A68

Van Eck, S., \& Jorissen, A. 2000, A\&A, 360, 196

Van Eck, S., Jorissen, A., Udry, S., Mayor, M., \& Pernier, B. 1998, A\&A, 329, 971

Van Eck, S., Neyskens, P., Jorissen, A., et al. 2017, A\&A, 601, A10

Wallerstein, G., \& Dominy, J. F. 1988, ApJ, 330, 937

Wang, X. H., \& Chen, P. S. 2002, A\&A, 387, 129

Wenger, M., Ochsenbein, F., Egret, D., et al. 2000, A\&AS, 143, 9

Yang, X., Chen, P., Wang, J., \& He, J. 2006, AJ, 132, 1468 


\section{Appendix A: Spectral energy distributions of the sample stars}

Table A.1. Elemental abundances of the two bitrinsic candidates, along with the standard deviation due to line-to-line scatter.

\begin{tabular}{|c|c|c|c|c|c|c|c|c|c|c|c|c|}
\hline & \multirow[b]{2}{*}{$\mathrm{Z}$} & \multirow[b]{2}{*}{$\log \epsilon_{\odot}$} & \multicolumn{5}{|c|}{$o^{1}$ Ori } & \multicolumn{5}{|c|}{$\mathrm{BD}+79^{\circ} 156$} \\
\hline & & & $\log \epsilon$ & $N$ & {$[\mathrm{X} / \mathrm{H}]$} & {$[\mathrm{X} / \mathrm{Fe}]$} & $\sigma_{[\mathrm{X} / \mathrm{Fe}]}$ & $\log \epsilon$ & $N$ & {$[\mathrm{X} / \mathrm{H}]$} & {$[\mathrm{X} / \mathrm{Fe}]$} & $\sigma_{[\mathrm{X} / \mathrm{Fe}]}$ \\
\hline $\mathrm{C}$ & 6 & 8.43 & 8.23 & & -0.19 & 0.09 & 0.10 & 8.36 & & -0.07 & 0.09 & 0.17 \\
\hline $\mathrm{N}$ & 7 & 7.83 & $8.20 \pm 0.13$ & & 0.37 & 0.65 & 0.65 & $8.70 \pm 0.13$ & & 0.87 & 1.04 & 0.65 \\
\hline $\mathrm{O}$ & 8 & 8.69 & 8.36 & & -0.33 & -0.05 & & 8.66 & & -0.03 & 0.14 & \\
\hline $\mathrm{Fe}$ & 26 & 7.50 & $7.21 \pm 0.19$ & 13 & -0.28 & - & 0.23 & $7.33 \pm 0.12$ & 13 & -0.17 & - & 0.18 \\
\hline Y I & 39 & 2.21 & $1.90 \pm 0.00$ & 1 & -0.31 & -0.02 & 0.27 & $2.80 \pm 0.00$ & 1 & 0.59 & 0.76 & 0.29 \\
\hline Y II & 39 & 2.21 & $2.30 \pm 0.00$ & 1 & 0.09 & 0.37 & 0.27 & - & - & - & - & - \\
\hline Zr I & 40 & 2.58 & $2.45 \pm 0.07$ & 2 & -0.13 & 0.15 & 0.12 & $3.25 \pm 0.07$ & 2 & 0.67 & 0.84 & 0.12 \\
\hline $\mathrm{Nb} \mathrm{I}$ & 41 & 1.46 & $1.30 \pm 0.15$ & 3 & -0.16 & 0.12 & 0.18 & $2.10 \pm 0.00$ & 3 & 0.64 & 0.81 & 0.14 \\
\hline $\mathrm{Tc}$ & 43 & & $-0.4 \pm 0.00$ & 1 & & & 0.17 & $-0.7 \pm 0.00$ & 1 & & & 0.17 \\
\hline $\mathrm{Ba} \mathrm{I}$ & 56 & 2.18 & $1.60 \pm 0.00$ & 1 & -0.58 & -0.29 & 0.17 & $2.60 \pm 0.00$ & 1 & 0.42 & 0.59 & 0.17 \\
\hline Ce II & 58 & 1.58 & $1.25 \pm 0.28$ & 6 & -0.33 & -0.04 & 0.17 & $1.96 \pm 0.28$ & 7 & 0.38 & 0.55 & 0.24 \\
\hline Pr II & 59 & 0.72 & $1.05 \pm 0.21$ & 2 & 0.33 & 0.61 & 0.23 & $1.50 \pm 0.00$ & 1 & 0.78 & 0.95 & 0.14 \\
\hline $\mathrm{Nd}$ II & 60 & 1.42 & $1.37 \pm 0.15$ & 5 & -0.04 & 0.24 & 0.26 & $2.10 \pm 0.00$ & 2 & 0.68 & 0.85 & 0.14 \\
\hline Sm II & 62 & 0.96 & $0.85 \pm 0.10$ & 2 & -0.11 & 0.17 & 0.14 & $1.30 \pm 0.00$ & 1 & 0.34 & 0.51 & 0.14 \\
\hline Eu II & 63 & 0.52 & - & - & - & - & - & $0.85 \pm 0.00$ & 1 & 0.33 & 0.50 & 0.12 \\
\hline
\end{tabular}

Notes. Solar abundances (third column) are from Asplund et al. (2009). The column labeled $N$ lists the number of lines used to derive the corresponding elemental abundance. The $\sigma_{[\mathrm{X} / \mathrm{Fe}]}$ column lists the total uncertainty on the abundance calculated using the method described in Sect. 4.
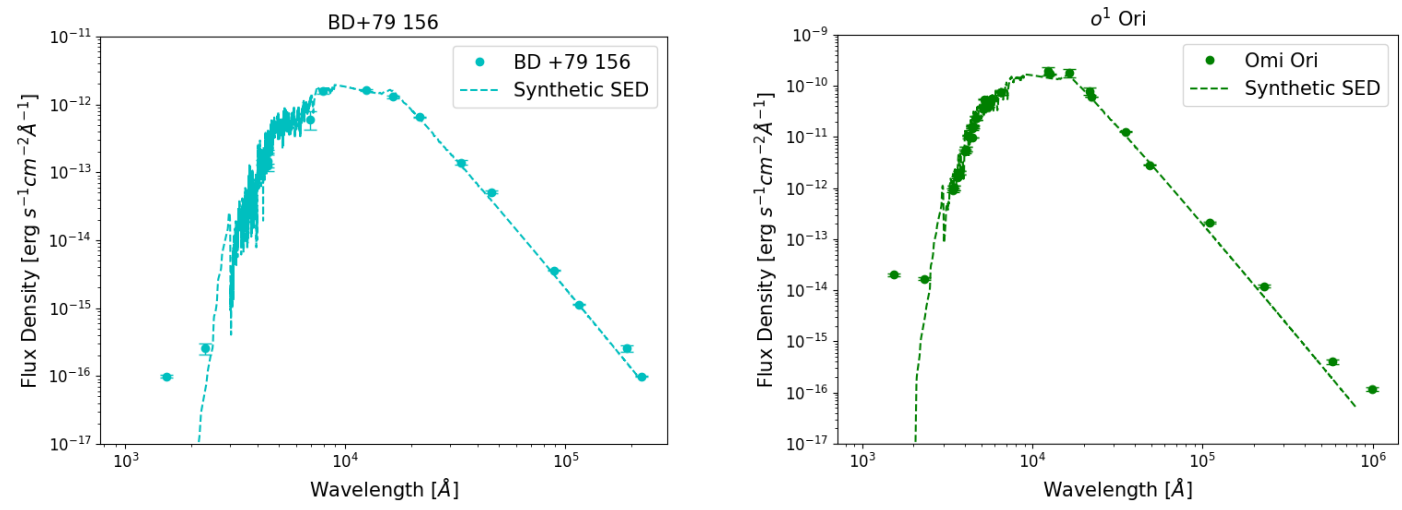

Fig. A.1. Photometric observations of $\mathrm{BD}+79^{\circ} 156$ (left panel) and $o^{1}$ Ori (right panel) compared with the synthetic spectral energy distribution generated using the MARCS model atmospheres with the parameters listed in Table 1. All photometric observations were retrieved from SIMBAD (Wenger et al. 2000). We note that in the two stars, the evident UV excess is in the bluest flux point.

Table A.2. Atomic lines used in this study.

\begin{tabular}{lcccc}
\hline \hline Species & $\lambda[\AA]$ & $\chi[\mathrm{eV}]$ & $\log g f$ & Reference \\
\hline Fe I & 7389.398 & 4.301 & -0.460 & Kurucz (2007) \\
& 7418.667 & 4.143 & -1.376 & O'Brian et al. (1991) \\
& 7443.022 & 4.186 & -1.820 & Martin et al. (1988) \\
& 7461.263 & 5.507 & -3.059 & Kurucz (2007) \\
& 7498.530 & 4.143 & -2.250 & Martin et al. (1988) \\
& 7540.430 & 2.727 & -3.850 & Martin et al. (1988) \\
& 7568.899 & 4.283 & -0.773 & Kurucz (2007) \\
& 7583.787 & 3.018 & -1.885 & O'Brian et al. (1991) \\
& 7586.018 & 4.313 & -0.458 & Kurucz (2007) \\
& 7832.196 & 4.435 & 0.111 & Kurucz (2007) \\
& 8108.320 & 2.728 & -3.898 & Kurucz (2007) \\
& 8698.706 & 2.990 & -3.452 & Kurucz (2007) \\
& 8699.454 & 4.955 & -0.380 & Nave et al. (1994) \\
& 8710.404 & 5.742 & -5.156 & Kurucz (2007) \\
Zr I & 8729.144 & 3.415 & -2.871 & Kurucz (2007) \\
& 7819.374 & 1.822 & -0.380 & Biémont et al. (1981) \\
Nb I & 7849.365 & 0.687 & -1.300 & Biémont et al. (1981) \\
& 5189.186 & 0.130 & -1.394 & Duquette \& Lawler (1982) \\
& 5271.524 & 0.142 & -1.240 & Duquette \& Lawler (1982) \\
\hline
\end{tabular}

